\title{
Impaired RNA splicing of 5'-regulatory sequences of the astroglial glutamate transporter EAAT2 in human astrocytoma
}

\author{
C Münch, A Penndorf, B Schwalenstöcker, D Troost, A C Ludolph, P Ince, ${ }^{\star}$ T Meyer
}

\begin{abstract}
A loss of the glutamate transporter EAAT2 has been reported in the neoplastic transformation of astrocytic cells and astrocytoma. The RNA expression of EAAT2 and five 5'-regulatory splice variants was investigated to identify alterations of the post-transcriptional EAAT2 gene regulation in human astrocytic tumours.
\end{abstract}

Three known (EAAT2, HBGTII, and HBGTIIC) and two novel (EAAT2/3 and EAAT2/31) EAAT2 transcripts originating from alternative splicing of 5'-regulatory sequences were subject to an RNA expression analysis using reverse transcription and competitive PCR. Specimens of astrocytoma World Health Organisation (WHO) grade I-IV in 14 patients and control brain tissue obtained from three normal persons were studied.

Neurology, Building O25, Room 5101, University of Ulm, Albert-Einstein Allee 11, 89081 Ulm, Germany

C Münch

A Penndorf

B Schwalenstöcker

A C Ludolph

T Meyer

MRC Neurochemical Pathology Unit, Newcastle General Hospital, Newcastle upon Tyne, UK

P Ince

Department of Pathology, Academic Medical Center, University of

Amsterdam, The Netherlands

D Troost

Correspondence to: Dr T Meyer

thomas.meyer@

medizin.uni-ulm.de

Received 3 September 2000 and in revised form

4 April 2001

Accepted 18 July 2001

$\star$ These authors contributed equally.
The main EAAT2 RNA was found to be equally expressed in normal human brain and astrocytic tumour samples. By contrast, the expression pattern of four 5 '-variants of the transporter transcript was altered in the investigated series of astrocytoma compared with normal brain. HBGTII, HBGTIIC, and EAAT2/3 were amplified from seven and four tumours and one sample, respectively. EAAT2/31 was expressed in none of the tumour specimens studied.

In conclusion, in astrocytic tumours of different histopathological grades there was a substantial reduction of RNA splicing events in EAAT2. The impairment of EAAT2 splicing indicates an altered expression which is not primarily involved in the tumorigenesis but may contribute to some biological properties of astrocytoma such as oedema, necrosis, and tumour related seizures.

(F Neurol Neurosurg Psychiatry 2001;71:675-678)

Keywords: glutamate transporter; RNA; splicing; astrocytoma

Glutamate is the major excitatory neurotransmitter of the human brain. Furthermore, it is a potent neurotoxin leading to glutamate mediated damage of postsynaptic neurons via excitotoxic mechanisms. High affinity glutamate transporter serves to maintain extracellular glutamate below excitotoxic concentrations. ${ }^{1}$ The astroglial excitatory amino acid transporter 2 (EAAT2) is the main protein of glutamate uptake. ${ }^{2}$ A defective expression of the EAAT2 protein has recently been reported in human glioma cells and astrocytic tumours. ${ }^{3}$ The compromised glutamate uptake of glioma cells has been suggested to contribute to tumour associated necrosis and seizures. ${ }^{3}$ In previous studies we found a complex posttranscriptional RNA regulation of EAAT2 resulting from alternative splicing of protein coding and untranslated exons, and differential RNA cleavage and polyadenylation. ${ }^{4-7}$ We cloned four EAAT2 splice forms of coding sequences which most likely encode nonfunctional EAAT2 polypeptides. ${ }^{45}$ For one of these transcripts a loss of function and a negative dominant effect on transporter expression has been shown. ${ }^{8}$ Several alternative transcripts of EAAT2 were characterised by diverse and different 5'-untranslated sequences and $N$-termini of the putative EAAT2 polypeptide. $^{9-11}$ Thus, splicing events of the EAAT2 RNA resulted in three alternative translational start sites leading to variable lengths of the transporter $N$-terminus by nine and five amino acids, respectively. In this study we report the expression analysis of three previously published (EAAT2, HBGTII, HBGTIIC) and two novel 5'-splice variants (EAAT2/3, EAAT2/31) of the EAAT2 transcript in human astrocytic tumours. ${ }^{9-11}$

\section{Materials and methods}

TISSUE SAMPLES

Brain tissue samples that were not required for diagnostic purposes of pilocystic astrocytoma (World Health Organisation (WHO) I; $\mathrm{n}=4$ ), low grade astrocytoma (WHO II; $n=4$ ), anaplastic astrocytoma (WHO III; $n=2$ ) and glioblastoma multiforme (WHO IV; n=4) were obtained from the Newcastle brain tissue bank, Newcastle upon Tyne, UK. The tissue, taken from biopsy, was snap frozen and stored at -80 ${ }^{0} \mathrm{C}$ until use. Control RNA samples of three non-neurological patients were purchased 
from a commercial source (human cerebral cortex, hippocampus, cerebellum, thalamus cDNA, Invitrogen).

POLYMERASE CHAIN REACTION CLONING

To identify novel 5'-splice forms of EAAT2 we performed a rapid amplification of 5'-cDNA ends (RACE) using human whole brain cDNA ligated with a 5'-adaptor sequence (Clontech).
The 5'-ends of the EAAT2 RNA were amplified by polymerase chain reaction (PCR) using adaptor (Clontech) and a 5'-gene specific primer (5'-GAAGGCTATTAACATAACCA CATC). ${ }^{9}$ The cDNA was passed through five cycles of PCR amplification at $94^{\circ} \mathrm{C}(45 \mathrm{~s})$ and $70{ }^{\circ} \mathrm{C}$ ( 4 minutes) followed by 25 cycles at $94^{\circ} \mathrm{C}$ (20 s) and $68{ }^{\circ} \mathrm{C}$ (4 minutes), respectively. The resulting product was a template of a second
A

EAAT2

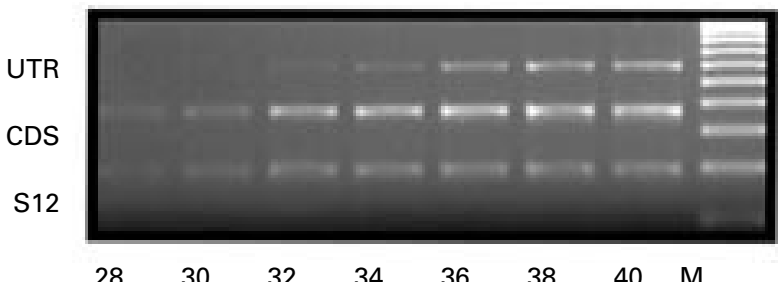

HBGTII

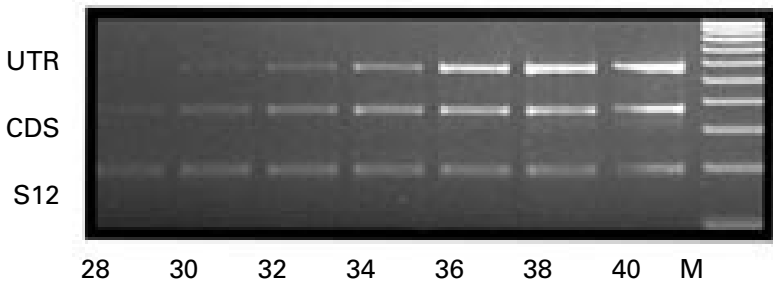

HBGTIIC

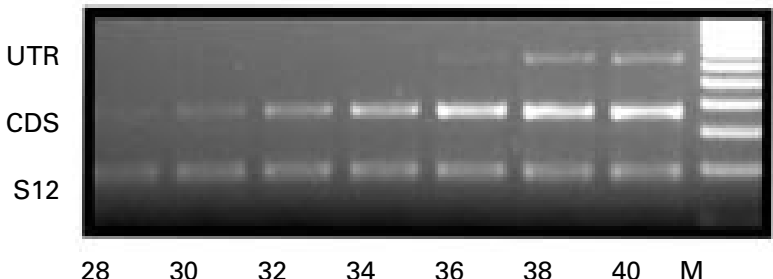

EAAT2/3

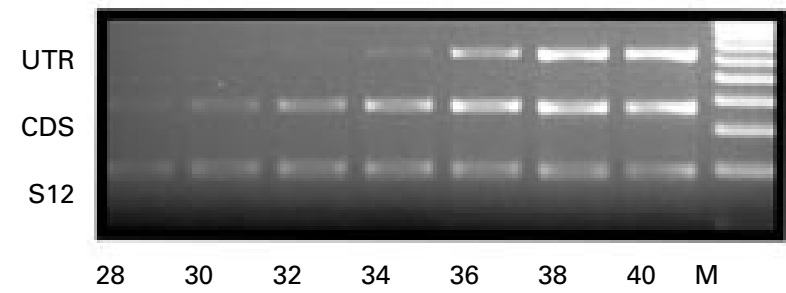

EAAT2/31

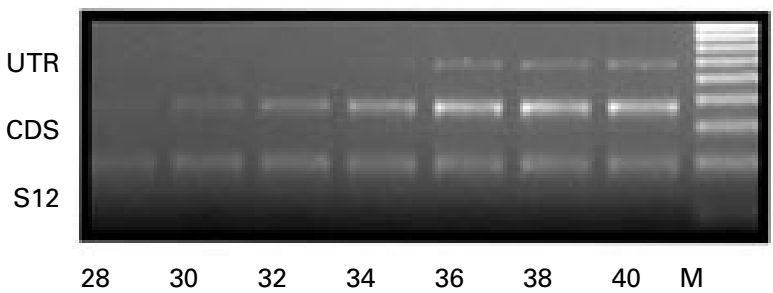

EAAT2

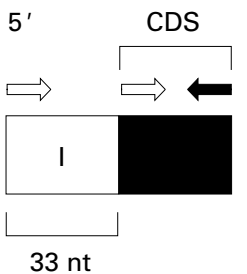

HBGTII

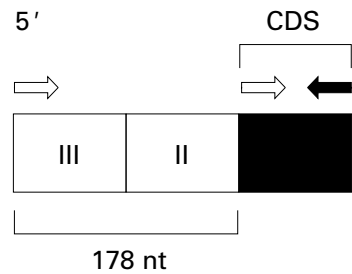

$5^{\prime}$

HBGTIIC

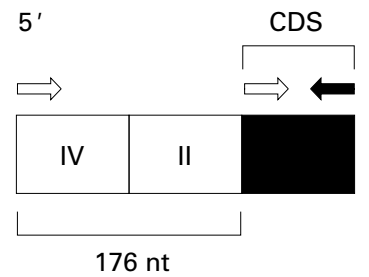

EAAT2/3

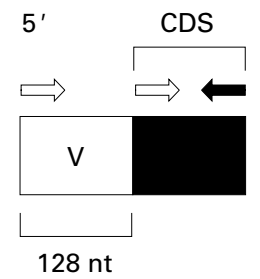

EAAT2/31

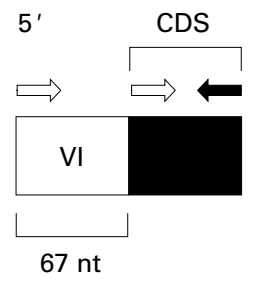

Figure 1 Schematic representation of the structure and expression of 5'-regulatory variants of the EAAT2 RNA in the normal human brain. (A) Competitive PCR of five EAAT2 transcripts (UTR) in the normal human cerebral cortex as coamplified with an internal standard of the EAAT2 RNA (CDS) and a home keeping gene $S 12 \mathrm{mRNA}$. PCR products obtained from cycle 28 to 40 were subjected to agarose gel electrophoresis and visualised by ethidium bromide staining. $n t=n u c l e o t i d e s ; U T R=u n t r a n s l a t e d ~ r e g i o n ; ~ C D S=$ coding sequence. (B) Five EAAT2 transcripts resulting from alternative splicing of six 5'-exons which encode 5'-untranslated and the N-terminal protein coding sequences. The 5'-exons are displayed in plain boxes and are numbered I-VI. The coding part (CDS) is symbolised by a black box. Arrows indicate the position of primers used for PCR analysis. 
amplification using nested adapter (Clontech) and gene specific primers (5'-CAGGCGCAG GCCCAGGTG).$^{9}$ The amplification product was subcloned (pCR2.1, TA cloning kit, Invitrogen) and insert containing constructs were sequenced (ABI 377 DNA sequencer, Applied Biosystems). Total RNA from $50 \mathrm{mg}$ of each tumour sample was isolated as described by the manufacturer (Micro RNA isolation kit, Stratagene). The yield of total RNA isolation was analyzed by quantitative densitometry using a computerised analysis program (ImageQuant Software, Molecular Dynamics). Equal amounts of $1 \mu \mathrm{g}$ of total RNA were used as template for the reverse transcription. The primary strand synthesis of complementary DNA was based on random priming (random hexamers, GeneAmp, Perkin Elmer) and carried out according to the protocol of the manufacturer (GeneAmp, Perkin Elmer) for 15 minutes at $42{ }^{\circ} \mathrm{C}$. Competitive PCR was performed as multiplex PCR of a splice form specific amplicon (EAAT2, HBGTII, HBGTIIC, EAAT2/3 and EAAT2/31), of an amplification product of the coding sequence (CDS) and of an internal standard (40S ribosomal protein $\mathrm{S} 12 \mathrm{mRNA}$ ). We designed primers complementary to the 5'-untranslated sequences of the specific transcripts (EAAT2 5'-GAAGAGGAGGGGCGTTCCC; HBGTII 5'-GGACCTGGGACCCCCAGAC; HBGTIIC 5'-GTGGTGTGCCTGCCTGT GAG; EAAT2/3 5'-GGAAGTGAATCTGA GACCTGTC; EAAT2/31 5'-GAGGTCAG CAGGAGGAAGAC; CDS 5'-AGGCTTGG ACAAGGTTTTCAGGG) (fig 1). The PCR product EAAT2/CDS was localised to a part of the protein coding sequence of EAAT2 (nt 1282-1593) which is not subject to alternative splicing and preserved in all EAAT2 transcripts known to date. ${ }^{9}$ EAAT2/CDS therefore represents the total amount of constitutively and alternatively spliced EAAT2 transcripts and served as an expression standard for coamplification of individual 5'-splice variants of EAAT2. The multiplex PCR was carried out for 40 cycles of denaturation at $94^{\circ} \mathrm{C}(15 \mathrm{~s})$, annealing at $60{ }^{\circ} \mathrm{C}(30 \mathrm{~s})$ and extension at $72^{\circ} \mathrm{C}$ (45 s) (AmpliTaq, Perkin Elmer). $5 \mu$ of PCR reaction products were removed after the cycles $28,30,32,34,36,38$, and 40 separated on $2.5 \%$ agarose gel and stained with ethidium

Table 1 Expression of five 5'-splice variants of EAAT2 in astrocytic tumours

\begin{tabular}{lllllll}
\hline \multirow{2}{*}{$\begin{array}{l}\text { Tumour } \\
\text { Patient }\end{array}$} & \multicolumn{5}{l}{ Glutamate transporter splice forms } \\
\cline { 3 - 7 } & (WHO) & EAAT2 & HBGTII & HBGTIC & EAAT2/3 & EAAT2/31 \\
\hline 1 & I & H & M & 0 & 0 & 0 \\
2 & I & H & 0 & 0 & 0 & 0 \\
3 & I & H & H & 0 & 0 & 0 \\
4 & I & H & 0 & 0 & 0 & 0 \\
5 & II & H & H & L & M & 0 \\
6 & II & H & 0 & 0 & 0 & 0 \\
7 & II & H & H & 0 & 0 & 0 \\
8 & II & H & L & L & 0 & 0 \\
9 & III & H & 0 & 0 & 0 & 0 \\
10 & III & H & 0 & L & 0 & 0 \\
11 & IV & H & M & 0 & 0 & 0 \\
12 & IV & H & M & 0 & 0 & 0 \\
13 & IV & H & 0 & L & 0 & 0 \\
14 & IV & H & 0 & 0 & 0 & 0 \\
\hline
\end{tabular}

$\mathrm{H}=$ High expression compared with S12mRNA; $M=$ medium; $\mathrm{L}=$ low; 0 = not amplifiable. bromide. Each of the multiplex PCR amplifications were reproduced three times. Images were captured by UV translumination (GelDoc1000, BioRad) and quantified (MultiAnalyst Software, BioRad). Comparative expression analysis of EAAT2 transcripts was performed during linear regression of amplification in relation to the signal of the internal standard RNA.

\section{Results}

In the human brain variable EAAT2 transcripts originating from alternative splicing of 5'regulatory sequences have been described. In this study we identified two previously unknown 5'-isoforms of the EAAT2 RNA. The novel transporter transcripts, named EAAT2/3 and EAAT2/31, were characterised by different 5 '-untranslated sequences showing low homology to previously reported EAAT2 transcripts. EAAT2/3 and EAAT2/31 encoded an open reading frame presenting a truncation of the $N$-terminus of the putative EAAT2 polypeptide by 5 amino acids. The $N$-terminus of both transcripts is identical to the HBGTII RNA and resulted from alternative splicing of 5 '-exons and differential usage of translational start codons. ${ }^{6}$

For all of the transcripts in the three normal controls we found a regional expression pattern in the cortex $>$ hippocampus $>>$ thalamus and cerebellum. The tissue samples included in this study were obtained from supratentorial cortical and subcortical locations. In the normal human cortex, the transporter transcripts showed high (EAAT2, HBGTII, EAAT2/3), moderate (HBGTIIC), and low (EAAT2/31) expression levels (fig 1).

The expression analysis of the EAAT2 RNA and four alternative splice variants of EAAT2 (HBGTII, HBGTIIC, EAAT2/3 and EAAT2/ 31) showed a significant alteration of EAAT2 splicing events in the investigated series of astrocytic tumours (table 1). The expression of the constitutively spliced transporter RNA EAAT2 proved to be present in all tumour samples. By contrast, HBGTII and HBGTIIC were found in a reduced number of the tumour specimens $(50 \%$ and $29 \%$, respectively). EAAT2/3 was detected in a single tumour, whereas EAAT2/31 was expressed in none of the investigated astrocytic tumour specimens (table 1).

In tumour samples containing of HBGTII, HBGTIIC and EAAT2/3 the quantitative expression of these splice forms was reduced compared with controls. Interestingly, the relative expression of the constitutively spliced transcript EAAT2 was unchanged. The reduction (HBGTII, HBGTIIC, EAAT2/3) or loss (EAAT2/31) of splice variant expression was found in samples of low and high grade astrocytomas. We did not find a tumour location or stage related regulation of the alternatively spliced transporter transcripts. The internal standard 40S ribosomal protein S12 RNA was invariably amplified in the series of tumour tissues when equal amounts of total RNA were used as template for the reverse transcription and competitive PCR. The stable standard 
RNA expression proved the RNA intactness in the investigated collection of tumours and normal brain RNA samples.

\section{Discussion}

A reduced protein expression of the glutamate transporter EAAT2 and a diminished glutamate uptake have been reported in human astrocytoma, yet little is known about the regulation of the EAAT2 gene in astrocytic tumours. ${ }^{3}$ In the normal brain we identified a complex regulation of EAAT2 including alternative splicing of untranslated and coding sequences and differential cleavage/ polyadenylation. $^{4-711} \mathrm{~A}$ series of astrocytic tumours was investigated for the expression of five EAAT2 transcripts originating from alternative splicing of 5'-regulatory exons. The results indicate a substantial reduction of EAAT2 splicing events in astrocytic tumours leading to a lower number of splice variants expressed in neoplastic brain tissue. A loss of functional diversity of EAAT2 RNA regulation may be part of the neoplastic transformation of astrocytic cells. These findings contribute to the hypothesis that altered RNA splicing events are an important molecular mechanism during neoplastic evolution. ${ }^{12}$ The functional meaning of EAAT2 splicing regulation in astrocytomas has not been completely understood. From other genes it is known that differential 5 -untranslated sequences are involved in the regulation of RNA stability and localisation and translational control. ${ }^{13}$ Distinct splice variants of the EAAT2 RNA were found at different expression levels in a given brain region (fig 1). Furthermore, multiple tissue expression arrays showed a region specific expression pattern of alternatively spliced EAAT2 transcripts in the brain and outside the CNS (data not shown and Münch et $\left.a l^{7}\right)$. Under normal conditions, alternative splicing events are likely to relate to the topical expression of the EAAT2 protein. ${ }^{14}$ The functional impact of the astrocytoma related alterations of EAAT2 splicing on glutamate mediated neuronal damage is not completely delineated. However, it is conceivable that an impairment of glutamate transporter regulation may relate to distinct biological properties of astrocytic tumours such as oedema, necrosis and tumour related seizures. In this small series of specimens there was no clear correlation of the expression of distinct 5'-regulatory EAAT2 variants with the histopathologically defined tumour grades. In a larger series of astrocytoma it would be of interest to associate the EAAT2 splicing pattern with transporter protein expression, histopathological grading, and the clinical properties of astrocytic tumours.

In summary, in astrocytic tumours RNA splicing events of 5'-regulatory sequences of the astroglial glutamate transporter EAAT2 were found to be substantially reduced. We report a tumour related RNA splicing regulation in a gene which encodes a key molecule of excitatory neurotransmission and is not known to be primarily involved in tumorigenesis.

This work was supported by a grant from the VERUM foundation.

1 Nicholls D, Attwell D. The release and uptake of excitatory amino acids. Trends Pharmacol Sci 1990;11:462-8.

2 Tanaka K, Watase K, Manabe T, et al. Epilepsy and exacerbation of brain injury in mice lacking the glutamate transporter GLT-1. Science 1997;276:1699-702.

3 Ye ZC, Rothstein JD, Sontheimer H. Compromised glutamate transport in human glioma cells: reductionmislocalization of sodium-dependent glutamate transporters and enhanced activity of cystine-glutamate exchange. $\mathcal{F}$ Neurosci 1999;19:10767-77.

4 Meyer T, Münch C, Knappenberger B, et al. Alternative splicing of the glutamate transporter EAAT2 (GLT-1). Neurosci Lett 1998;241:68-70.

5 Meyer T, Fromm A, Münch C, et al. The RNA of the glutamate transporter EAAT2 is variably spliced in amyotrophic lateral sclerosis and normal individuals. F Neurol Sci 1999; 170:45-50.

6 Münch C, Schwalenstöcker B, Knappenberger B, et al. 5'-Heterogeneity of the human excitatory amino acid 5'-Heterogeneity of the human excitatory amino acid
transporter cDNA EAAT2 (GLT-1). Neuroreport 1998;9: transport

7 Münch C, Schwalenstöcker B, Hermann C, et al. Differential RNA cleavage and polyadenylation of the glutamate transporter EAAT2 in the human brain. Brain Res Mol Brain Res 2000;80:244-51.

8 Lin CL, Bristol LA, Jin L, et al. Aberrant RNA processing in a neurodegenerative disease: the cause for absent EAAT2, a glutamate transporter, in amyotrophic lateral sclerosis. Neuron 1998;20:589-602.

9 Arriza LJ, Fairman WA, Wadiche JI, et al. Functional comparisons of three glutamate transporter subtypes cloned from human motor cortex. I Neurosci 1994;14:5559-69.

10 Shashidharan P, Wittenberg I, Plaitakis A, et al. Molecular cloning of human brain glutamate/aspartate transporter II. cloning of human brain glutamate/aspar
Biochim Biophys Acta 1994;1191:393-6.

11 Meyer T, Speer A, Meyer B, et al. The glial glutamate transporter complementary DNA in patients with amyotrophic porter complementary DNA in patients with

12 Cooper DL, Dougherty GJ, To metastasize or not? Selection of CD44 splice sites. Nat Med 1995;1:635-7.

13 Smith CW, Valcarcel J. Alternative pre-mRNA splicing: the ogic of combinatorial control. Trends Biochem Sci 2000;25: 381-8.

14 Milton ID, Banner SJ, Ince PG, et al. Expression of the glial glutamate transporter EAAT2 in the human CNS: an immunohistochemical study. Brain Res Mol Brain Res 1997;52:17-31. 